\title{
The Thermal Analysis of Polyurethane Prodrug with Different Drug Content
}

\author{
Zhiguo Wen ${ }^{1, a^{*}}$, Chong $\operatorname{Tian}^{1, \mathrm{~b}}$ and Wanli Nie1 ${ }^{\mathrm{c}}$ \\ ${ }^{1}$ No. 778, BinHe Road, Shizhong District, Leshan, SiChuan, China, 614000 \\ awenzhiguo100@163.com, bianchong30@163.com, ${ }^{\mathrm{a}}$ niewl126@126.com
}

\begin{abstract}
Keywords: polyurethane; microphase separation; drug content; DSC; TG
Abstract. Polyurethane prodrugs with different drug contents were synthesized using polycaprolactone, hexamethylene diisocyanate and ciprofloxacin. The thermal analysis of the materials was carried out by DSC and TG. Results showed that the crystallinity of the materials reduced as the drug contents increased and the materials were thermostable to be sterilized for the medical use.
\end{abstract}

\section{Introduction}

Polyurethane (PU) is a kind of polymer materials synthesized by macromolecular polyol, isocyanate and chain extender like glycol or diamine. Polyol is called soft segment (SS), while the combination of isocyanate and chain extender is called hard segment(HS). Neighbouring HSs associate with each other because of their large cohesive energy and form a domain. The domains are dispersed in the SS. This phenomenon is so-called microphase separation. The situation of microphase separation directly impacts the physical and mechanical performance of PU materials. The HS's and SS's structure, the content of HS and the thermal history of PU are considered as main factors that affect the situation of micphase separation in PU.

PU prodrug(CFPU) was synthesized using polycaprolactone(PCL1250), hexamethylene diisocyanate(HDI) and ciprofloxacin(CF) as chain extender. The influence of drug content on the thermodynamic properties of PU prodrug was studied by Differential scanning calorimetry analysis(DSC) and thermogravimetric analysis(TG). Drug content is not only relevant to the HS content, but also an important indicator of PU prodrug. So the drug content is a substitution of HS content to be considered.

\section{Experiment}

Reagents and raw materials. Polycaprolactone diol (PCL), average weight 1250 , and 1, 6-hexane diisocyanate (HDI) were obtained from the Aldrich Chemical Company. Ciprofloxacin hydrochloride (CF) powder was supplied by Jingxin Pharm. (Xinchang, Zhejiang, China) Dimethylsulphoxide (DMSO), tetrahydrofuran (THF), N, N-dimethylformamide (DMF), dibutyltin dilaurate (DBTDL) and triethylamine (TEA) were all gained from Sinopharm Chemical Reagent CO. , Ltd.

PU prodrug synthesis. The drug polymer was synthesized via a two-step solution polymerization reaction. The stoichiometry of the polymerization is 1: 0.66: 0.34 of HDI: PCL: CF.

Prepolymerization. PCL was dissolved and stirred in $50 \mathrm{~mL}$ DMSO at $60^{\circ} \mathrm{C}$ in a three-neck flask. The whole system was in a dehydrated nitrogen atmosphere. Then equimolar HDI and two droplets of DBTDL were added into the solution. The reaction took $3 \mathrm{~h}$.

Chain Extension. After prepolymerization, CF was added into the solution and TEA was added to neutralize the hydrochloric acid contained in the CF. HDI, with equal molar ratio to CF, was added subsequently. The solution was stirred at $60^{\circ} \mathrm{C}$ for $24 \mathrm{~h}$.

Eventually, the reaction was ended by the addition of $5 \mathrm{~mL}$ methanol and stirred for another hour to terminate the residual -NCO group. The reagent was cooled down and precipitated in a mixture of ether and distilled water. The precipitate was stirred in distilled water for over 3 days, while the water 
was intermittently changed each day. The polymer was dried in a convection oven at $40^{\circ} \mathrm{C}$ for $24 \mathrm{~h}$, and subsequently vacuum dried at $40^{\circ} \mathrm{C}$ for another $24 \mathrm{~h}$.

Drug contents of the prodrugs. Tab.1 shows the drug contents of CFPUs.

Tab.1 molar ratio of raw materials and drug contents of CFPUs

\begin{tabular}{cccc}
\hline sample & Molar ratio & Theoritical drug & HH contents \\
& (HDI: PCL: CF· HCl) & contents/\% & 14.7 \\
CFPU-1 & $1: 1: 0$ & 0 & 19.8 \\
CFPU-2 & $1: 0.78: 0.22$ & 6 & 34.8 \\
CFPU-3 & $1: 0.5: 0.5$ & 17.3 & 51.7 \\
CFPU-4 & $1: 0.3: 0.7$ & 30 & \\
\hline
\end{tabular}

Characterization. The DSC-TG analysis was carried out by STA449C, NETZSCH. The heating rate was $10^{\circ} \mathrm{C} / \mathrm{min}$. The carrier gas was argon argon, $30 \mathrm{~mL} / \mathrm{min}$.

\section{Results and discussion}

DSC analysis of CFPU. Different drug content means different SS/HS ratio, which affects glass transition temperature (Tg) and melt temperature(Tm) of PU prodrug. Fig.1 shows DSC curves of CFPU samples and PCL-1250. It's known that PCL's Tg and Tm temperature is about $-60^{\circ} \mathrm{C}$ and $60^{\circ} \mathrm{C}$, respectively. PCL has a superior crystallinity, which is weakened by microphase separation in CFPU because of the existence of hard segment. That makes the Tg and Tm of CFPUs different from PCL.

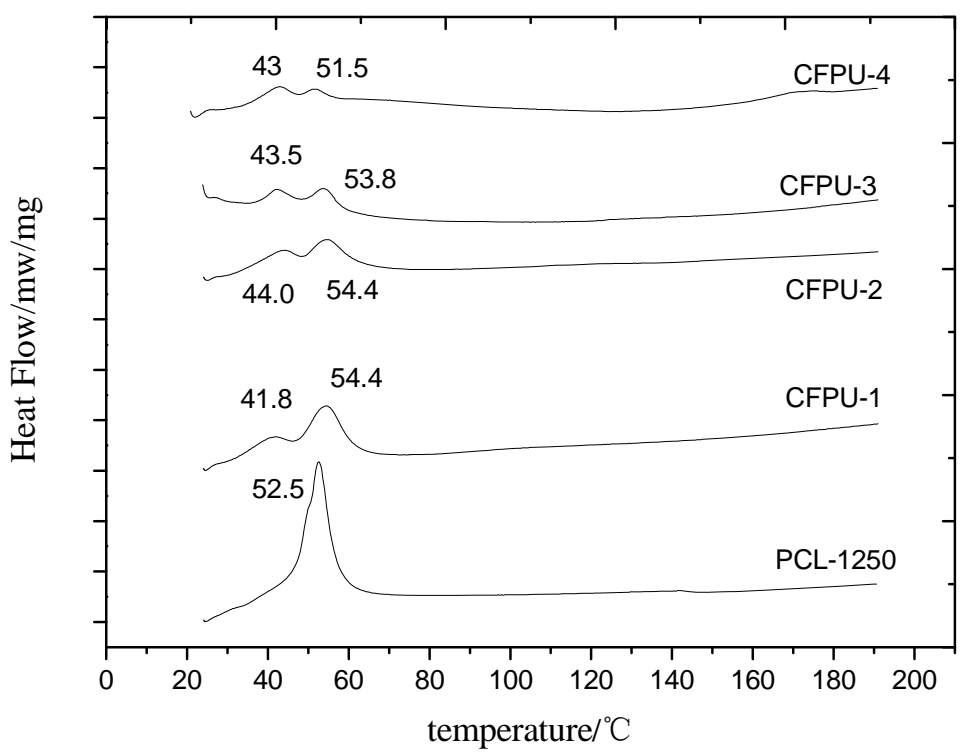

Fig. 1 DSC curves of CFPUs and PCL

(CFPU-1: 0; CFPU-2: 6\%; CFPU-3: 30\%; CFPU-4: 17.3\%)

Fig. 1 compared DSC curves of PCL-1250 and CFPUs with various drug contents. CFPU-1 was blank PU that contains no CF. It can be seen that PCL melt at $52.5^{\circ} \mathrm{C}$ and CFPUs had two endothermic peaks. Since the PCL's Tg is about $-60^{\circ} \mathrm{C}$ and Tm as Fig. 1 shows was $52.5^{\circ} \mathrm{C}$, it could be concluded that peaks at $41.5,43,46.5^{\circ} \mathrm{C}$ were HS's glass transition peaks. Temperature at about $41^{\circ} \mathrm{C}$ is the Tg temperature of HS. The contents of HS increased as the drug contents increased. Fig. 1 shows that the melt peaks of PCL(SS) weakened as the HS contents increased, the Tm of SS and Tg of HS also increased. The melt peaks of SS weakened means that crystallinity of CFPU reduced, which makes CFPU tensile and flexible.

TG and DTG analysis of CFPU. CFPU was designed for in vivo use. High temperature sterilization is a typical way to sterilize materials for in vivo use. Typically, the temperature use to sterilize biomedical materials is among $110 \sim 200^{\circ} \mathrm{C}$. So CFPUs is supposed to be thermostable at about $200^{\circ} \mathrm{C}$. 


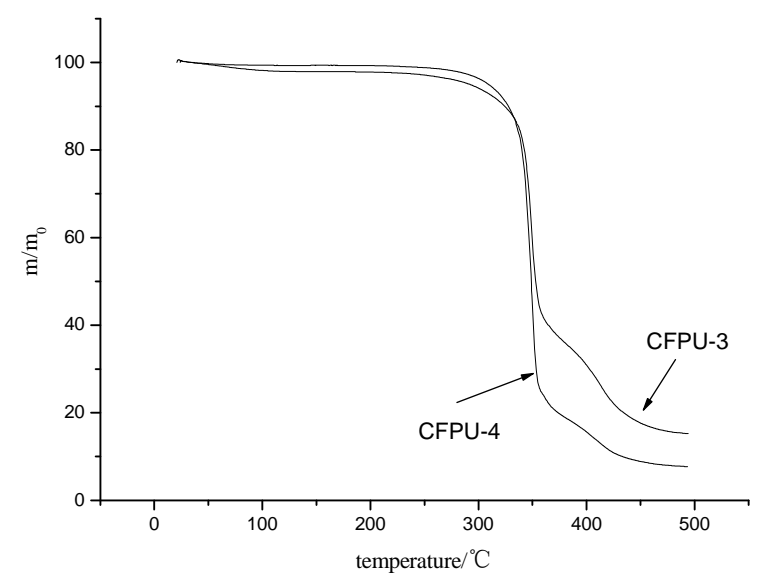

Fig.2 TG curves of CFPUs

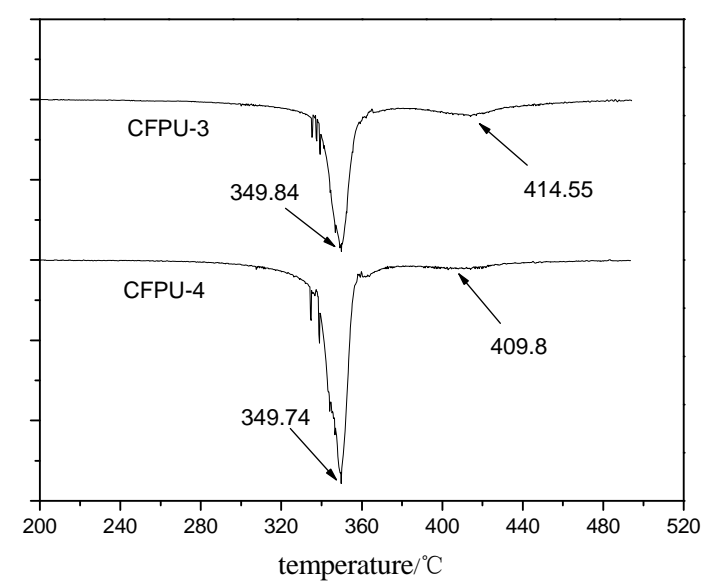

Fig.3 DTG curves of the CFPUs

Fig. 2 and Fig. 3 showed that CFPU3 and CFPU4 began to decompose at about $300^{\circ} \mathrm{C}$. This means CFPUs were thermostable to be sterilized by heat or high temperature steam.

\section{Conclusion}

A series of CFPUs were synthesized via two-step solution polymerization. Thermodynamic analysis of CFPUs and PCL was carried out. DSC experiments showed that, the crystallinity of CFPU reduced as the drug content increased. That makes CFPU more tensile and flexible. TG and DTG analysis confirmed that the material is thermostable and can be sterilized by high temperature sterilization to be used for medical use.

\section{Acknowledgements}

It is a project supported by Scientific Research Fund of Sichuan Provincial Education Department (Grant No. 15ZB0265) and Leshan Normal University (Grant No. Z1306). The author also acknowledges the supportance of Science and Technology Bureau of Leshan Town (Grant No. 14SZD011).

\section{References}

[1] Rasha A. Azzam, Sahar K. Mohamed, Rob Tol, Valja Everaert, Harry Reynaers, Bart Goderis. Polymer Degradation and Stability, Vol. 92 (2007), p. 1316-1325.

[2] Wu Li, Anthony J., Ryan, Ingrid K., Meier. Macromolecules, vol. 35 (2002), p. 5034-5042. 
[3] Xiaobin Zhao, Lei Du, Xiaoping Zhang and Jian Zheng. Polymer Materials Science and Engineering. vol. 18 (2003), p. 16-19, In Chinese.

[4] Chengshen Zhu. Structure analysis of Polymer (Second Edition). Science Press(Peijing), 2010. In Chinese.

[5] Yunfang Wang, Rumin Wang. China Adhesives. vol.17 (2008), p. 15-20. In Chinese.

[6] Jie Ou, Liying Tian, Xinling Wang. Journal of Functional Polymers. vol. 23 (2010), p. 160-165. In Chinese.

[7] Dezhu Ma. Structure and Performance of Polymers (Performance). Science Press(Peijing), 2013. In Chinese.

[8] Begum, Muneera, Siddaramaiah. Journal of Materials Science, vol. 39(2004), p. 4615-4623. 